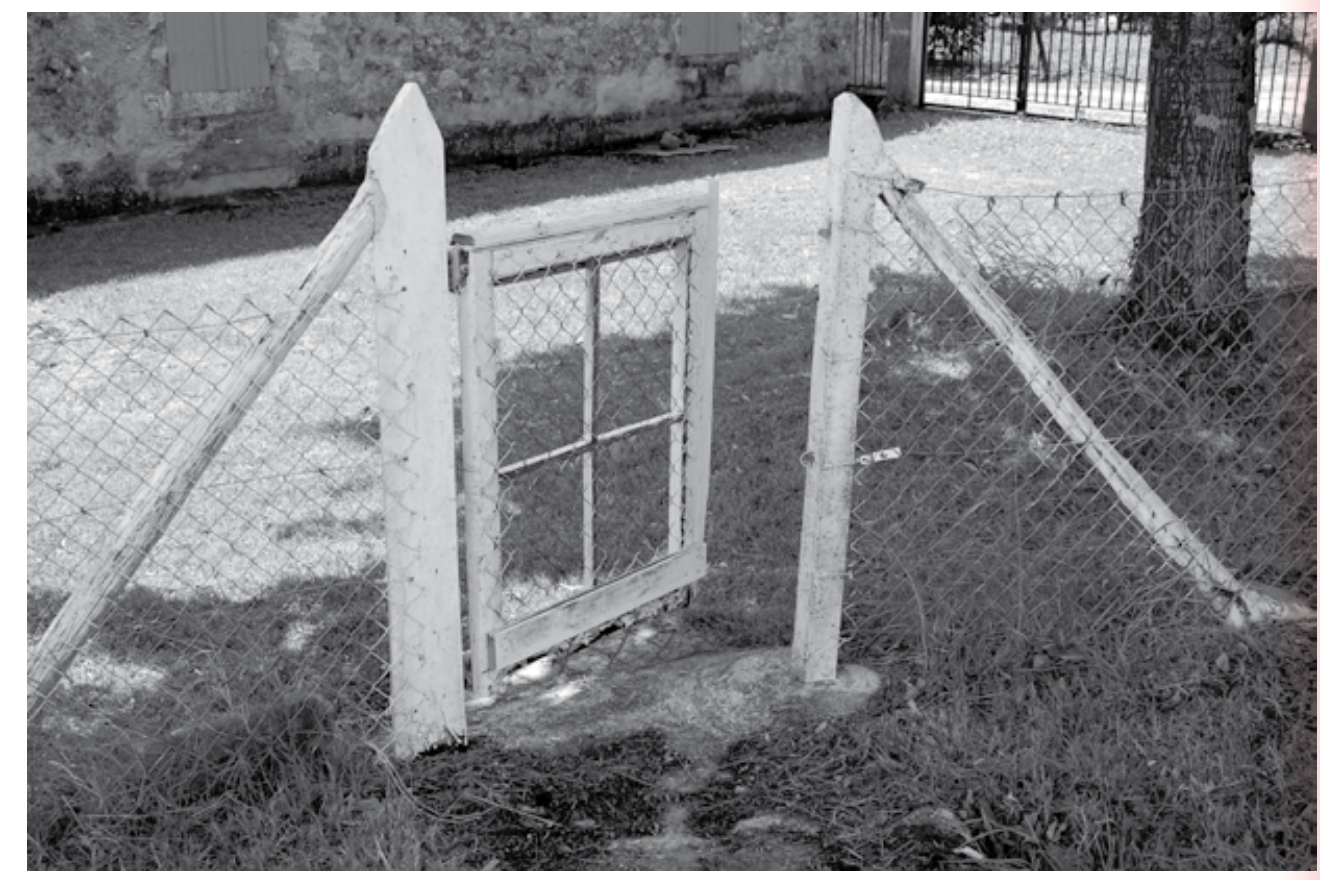

South West, France, 2007

\title{
Axiomes de survie pour une rythmanalyse politique
}

\author{
Yves Citton
}

Les propositions rythmologiques qui suivent sont formulées de manière dogmatique, en une série d'axiomes et de règles. Elles prolongent les deux axiomes (polyrythmie, microrythmie) articulés par Frédéric Bisson dans sa présentation d'Anna Cruz. Une telle axiomatique, ouverte à discussion, a valeur d'orientation générale pour l'intelligibilité de notre temps.

\section{La danse de la vie}

(synrythmie)

I. L'ensemble de notre vie sociale s'agence à travers une multitude de rythmes superposés, dont la plupart échappent à notre réflexion consciente. Nos comportements quotidiens forment une danse de la vie (Edward T. Hall), dont nous marquons incessamment le pas sans nous en rendre compte.

II. Tout rythme est collectif. Le rythme est agencement du multiple dans le multiple, et comme tel, il neutralise toute prétention à l'«autonomie». En notre époque de communication généralisée, il n'est de rythmes que synrythmiques: "mes» rythmes «personnels» sont forcément fonction de rythmisations collectives.
III. Le pouvoir dont se sent investie une subjectivité est en proportion directe de sa capacité à entraîner d'autres subjectivités dans ses rythmes propres. Edward T. Hall et William Condon parlent d'entraînement pour désigner la force d'attraction qui pousse une personne à synchroniser ses comportements avec ceux d'une autre personne. Tout rapport inter-humain met en contact des forces d'entraînement inégales.

IV. Les institutions sont des machines de synchronisation. La reproduction de nos existences socialisées implique donc une règle 1: plus les collaborations interhumaines gagnent en extension, plus les institutions qui doivent assurer la synchronisation de nos activités productives soumettent les agents à des hétérorythmies.

La résonance intérieure (endorythmie)

V. Tout rythme est endorythmique. Les rythmes sont vécus sous la forme de résonances intérieures: même dans les cas où on peut montrer qu'ils sont imposés de l'extérieur (hétérorythmie), leur dynamique doit nécessairement être réappropriée par le sujet, 
au point d'être perçue comme émanant de ments temporels bien marqués, accentués, lui. Un tempo peut s'imposer d'en haut; un donc contrastants, voire opposés comme rythme se vit de l'intérieur et doit toujours les temps forts et faibles», mais tout autant venir de l'agent. La puissance d'agir propre par des variations autour de ces scansions, au rythme vient donc du bas - non pas des ainsi que par «un mouvement d'ensemble «masses» (supposées homogènes), mais des qui emporte avec lui tous ces éléments (par singularités qui composent la multitude.

VI. Les rythmes (soutenables) résultent d'un double ajustement. Appartenir à une collectivité implique de pouvoir tout à la fois s'insérer dans les synchronisations qui la constituent, et y insuffler ses idiorythmies propres. Le rythme doit être soutenable et pour l'institution synchronisatrice et pour les agents qui collaborent en son sein: cela requiert de constants efforts d'ajustement réciproques. D'où une règle 2: plus les collaborations interhumaines s'intensifient, plus les institutions doivent être attentives aux idiorythmies des agents.

\section{Le rythme,}

c'est la tension d'un écart

VII. Le rythme danse autour de la périodi cité. Lorsque Pablo Casals affirme que «le rythme, c'est le retard», lorsque Henri Lefebvre et Catherine Régulier soulignent que «seul un mouvement non mécanique peut avoir un rythme», ils prennent le contrepied du lieu commun identifiant rythmicite et périodicité. Le rythme, en effet, consiste moins dans la répétition d'une même structure que dans les petits écarts imposés à la répétition métrique - c'est-à-dire dans ce que Pierre Sauvanet identifie comme le troisième élément du rythme (en plus de la structure et de la périodicité): le mouvement. «Le mouvement, c'est la forme mouvante opposée à la forme fixe, c'est le flux et cette «manière particulière de fluer» selon les «configurations particulières du mouvant» qu'évoque Benveniste». Le rythme se définit donc non seulement par «des élé-

\begin{abstract}
exemple un mouvement de valse, plus ou
\end{abstract} moins rapide)»(Henri Lefebvre et Catherine Régulier).

VIII. Le rythme consiste en une tension par rapport au retour périodique du mètre. La syncope qui anticipe un temps fort et la suspension qui le retarde ne sont que deux manières différentes de jouer avec des phénomènes de tension. Il y a par conséquent deux façons très différentes de saper la rythmicité: soit par l'effacement des marquages temporels (c'est l'«asthénie rythmique» dénoncée par Pascal Michon au cœur de la mondialisation néo-libérale); soit par l'imposition d'un marquage purement possible (hétérorythmie absolue). Ces deux écueils semblent cependant plus théoriques que réels: les sujets humains tendent toujours à réinsuffler leur endorythmie dans tout ce qu'ils font (même dans un univers dépourvu de scansions communes, même dans une chaîne de montage intégralement mécanisée). La tension du rythme accomà l'agir humain.

IX. Il ne saurait y avoir d'eurythmie à l'échelle de la société. Dès lors que le rythme consiste en la variation que l'agent impose de l'intérieur au retour périodique du mètre, il n'y a de «bon" rythme que dans le cadre du rapport entre cet agent singulier (individuel ou collectif) et cette activité. Le rythme s'affirme depuis le bas, par un déphasage (mineur) imposé à la norme commune. Il ne saurait y avoir de norme pour ce déphasage, pas plus qu'on ne saurait imposer depuis le haut un écart singularisant. mécanique, envers lequel aucun écart n'est pagne nécessairement la tension inhérente
Revendications

rythmiques

X. Il faut défendre un droit à l'écart rythmant. Une politique des rythmes ne doit pas seulement viser à réduire les tempi d'un monde dont Hartmut Rosa ou William Scheuerman soulignent à juste titre l'accélération effrénée; elle doit aussi dénoncer les hétérorythmies mutilantes dont le poids écrase le sujet (dont l'exemple classique est la chaîne de montage industrielle). Dès lors que la subjectivation passe par la capacité à infléchir les scansions communes selon la singularité des résonances intérieures propres à chacun, il convient d'assurer que les sujets (toujours en devenir) disposent des marges d'exécution nécessaires à l'affirmation de leur endorythmie propre.

XI. Il faut réclamer le droit d'imposer unilatéralement des rythmisations protectrices. Pascal Michon a raison de souligner les dangers liés à la dissolution de certains rythmes communs. Le courrier électronique constitue l'envers symétrique de la chaîne de montage: c'est parce qu'il n'impose aucune scansion temporelle commune qu'il exerce un poids anxiogène de plus en plus lourd sur nos subjectivités. Face à cette arythmicité numérique, non moins dommageable que l'hétérorythmicité industrielle, il faut se défendre en imposant unilatéralement ses rythmes propres aux pressions communicatrices: $j$ 'annonce que je ne regarde pas mes mails du samedi au mardi - ce qui, à partir d'une mesure rythmique, impose également une décélération au tempo de la communication.

XII. Il faut viser à la neutralisation des inégalités structurelles de la force d'entraînement. Que certains individus présentent une plus grande capacité à entraîner les autres dans leurs rythmes propres ne pose pas de problème en soi. En revanche, il faut veiller à compenser les inégalités et les asymétries directement générées par les positions institutionnelles - par exemple celles qui imposent aux subordonnés ou aux précaires un devoir de réponse instantanée, alors que le supérieur peut ignorer souverainement les requêtes qui lui sont adressées.

Revendications

polyrythmiques

XIII. Il faut diminuer les tensions polyrythmi ques insoutenables. Nous ne vivons jamais au sein d'un seul rythme, mais au sein de multiples couches rythmiques superposées, qui entrent fréquemment en tensions contradictoires entre elles (vie familiale, professionnelle, associative, activités sportives, intellectuelles, etc.). Aussi bien la micro-décision de prendre un appel téléphonique que la macro-décision de partir à la retraite s'inscrivent dans l'enchevêtrement de ces multiples couches, avec pour résultat que nos comportements ne sont jamais simplement rythmés, mais toujours polyrythmés. Dans les années 970, Edward T. Hall caractérisait le mode de vie européen et nord-américain comme étant monochrone, c'est-à-dire demandant aux individus de ne faire qu'une chose à la fois, par opposition aux cultures polychrones, dans lesquelles chacun est amené à jouer simultanément plusieurs rôles. Indépendamment des grandes catégorisations ethnologiques, on peut s'attendre à ce que (règle 3) plus les collaborations interhumaines se complexifient, plus se multiplient et s'exacerbent les tensions (frictions, tiraillements, entraînements contradictoires) entre les couches rythmiques superposées. En attendant que les générations successives apprennent à jouer simultanément dans plusieurs orchestres à la fois, une politique des rythmes doit également viser à mettre en place des isolants qui protègent les sujets d'écartèlements polyrythmiques destructurants. 
XIV. Il faut traiter la mondialisation comme un phénomène temporel aussi bien que géographique. Les divers phénomènes analysés au titre de la mondialisation ne paraissent souvent fluidifier (et dissoudre) les rythmes traditionnels que parce qu'ils surajoutent de nouvelles couches rythmiques qui brouillent ou désarticulent les couches inférieures. Les rythmes politiques (électoraux, budgétaires, fiscaux) des Étatsnations ont été récemment profondémen bouleversés par l'effondrement financie des subprimes nord-américains. Les télémarketeurs indiens doivent travailler nuit pour être synchrones avec la disponibilité des consommateurs occidentaux. Pascal Michon voit juste en se préoccupant des implications rythmiques de la mondialisation. Résister à leurs effets pervers est un déf majeur, qui ne peut se contenter de retrouver les rythmes d'un "bon vieux temps » illusoire et irrécupérable.

XV. Une politique des polyrythmes doit miser sur l'endorythmie, non tant pour "résis ter $»$ à la mondialisation que pour donner aux individus les moyens de la réinvestir de scansions signifiantes et de mouvements subjectivants. Dans la mesure où il repose sur un résonance intérieure (endorythmie) et dan la mesure où il consiste en une puissance de variation (écart), le rythme relève comme tel d'une puissance inaliénable. On peut casser mon rythme, on peut m'imposer un tempo on peut me synchroniser par l'entrainement d'une heterorythmie, mais c'est toujours du tréfonds de mon être qu'émane le rythme dont j'investis mon comportement. Sans jamais être "autonome», puisque les mouvements sont nécessairement synrythmiques, le rythme ne peut émaner que du sujet lui-même (tel qu'il se réapproprie sa relation au collectif). D'où une règle 4: plus la polyrythmie mondialisée nous pousse vers la polychronie, plus il est important de fortifier nos capacités de subjectivation. Edward T. Hall oppose la compartimentation disciplinaire sur laquelle reposent les systèmes monochrones à la relationalité inhérente aux systèmes polychrones, dont l'efficacité repose entièrement sur la «qualité humaine» des agents. En remettant «le capital humain» (dans ses dimensions relationnelles et affectives) au cour de la productivité contemporaine, les économistes reconnaissent implicitement que les institutions d'aujourd'hui et de demain ne peuvent être «gérées» que par des subjectivités fortement structurées - c'est-à-dire capables d'entraîner leur voisinage par la puissance de leur endorythmie. Il nous faudra sans doute apprendre très vite à inscrire quelque chose comme le sens $d u$ swing au rang des «fondamentaux» de l'éducation de demain, au même titre que la lecture ou le calcul.

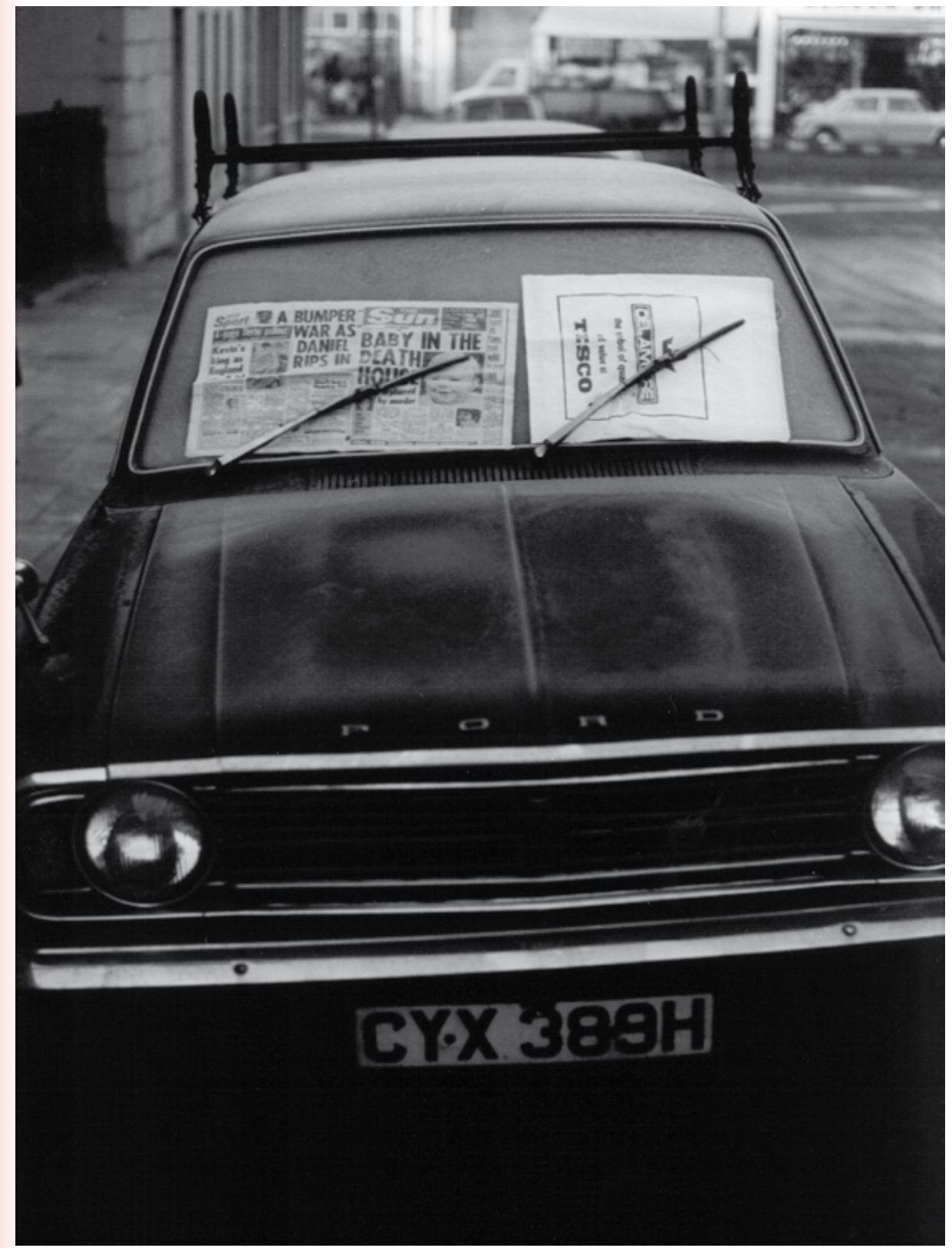

Caledonian Road, London, 1978 


\section{Bibliographie sommaire de rythmanalyse}

Nicolas Abraham, Rythmes: de la philosophie, de la psychanalyse et de la poésie, Paris, Flammarion, 1999

Gaston Bachelard, La dialectique de la durée, Paris, PUF, 1950, chapitre VIII, «La rythmanalyse»

Roland Barthes, Comment vivre ensem ble. Cours et séminaires au Collège de France 1976-1977, éd. Claude Coste, Paris, Seuil/ IMEC, 2002

Frédéric Bisson, «Entre le cristal et le brouillard. Rythme et Vie à partir de Whitehead», revue Chromatika, Annuaire de la philosophie en procès, $\mathrm{n}^{\circ} 5$, Presses universitaires de Louvain, 2009, pp. 25-42 (disponible en ligne sur rhuthmos.eu)

Frédéric Bisson, «Le swing cosmique. Whitehead à la mescaline», revue Chromatik $\mathrm{n}^{\circ}$ 5, Louvain, 2009, pp. 133-150 (disponible en ligne sur rhuthmos.eu)
Yves Citton, «Improvisation, rythmes et mondialisation. Quatorze thèses sur la fluidification sociale et les résistances idiorrythmiques», revue Textuel n'60, 2010, pp. 127-146 (disponible en ligne sur rhuthmos.eu, avec une réponse de Pascal Michon)

Anne-Claire Désesquelles, Au rythme de la vie, les éditions Ovadia, Nice, 2008

Maie Gérardot, «Rythmes des lieux, rythme de la ville», Urbanisme, janvier/février 2011, n³76

Matila C. Ghyka, Essai sur le rythme, Paris, Gallimard, 1938

Laurent Guido, L'Âge du rythme. Cinéma, musicalité et culture du corps dans les théories françaises des années 1910-1930, Lausanne, Payot, 2007

Edward T. Hall, La Danse de la vie. Temps culturel, temps vécu, Paris, Seuil, 1984
Olivier Hanse, À l'école du rythme. Utopies communautaires allemandes autour de 1900, Université de Saint-Étienne, 2010

Intermédialités, $\mathrm{n}^{\circ} 16$ «Rythmer/Rhythmize», dossier dirigé par Michael Cowan et Laurent Guido, automne 2010

Henri Lefebvre et Catherine Régulier, «Le projet rythmanalytique», Communications, 41, 1985, pp. 191-199

Henri Lefebvre, Éléments de rythmanalyse. Introduction à la connaissance des rythmes, Editions Syllepse, 1992

Henri Maldiney, «L'esthétique des rythmes", in. Regard, Parole, Espace, Lausanne, Lâge d'homme, 1973, pp.147-172

Alberto Melucci, «Rythmes internes et

Pascal Michon, Rythmes, pouvoir, mondialisation, Paris, PUF, 2005

Pascal Michon, Les Rythmes du politique. Démocratie et capitalisme mondialisés, Paris, Les Prairies ordinaires, 2007

Pascal Michon, «Idiorythmie ou eurythmie? Réponse à Yves Citton», en ligne sur http://rhuthmos.eu/spip.php?article94

RHUTHMOS, http://rhuthmos.eu/ (site entièrement dédié à la recherche et aux débats sur les rythmes)

Hartmut Rosa, Accélération. Une critique sociale du temps, Paris, La Découverte, 2010

Pierre Sauvanet, Le Rythme et la raison, Paris, Kimé, 2000 (2 volumes)

William E. Scheuerman, Liberal Demorythmes sociaux dans un monde planétaire», in. Nouvelles pratiques sociales, vol. $10, \mathrm{n}^{\circ} 2$, 1997, pp. 195-202 (disponible en ligne sur le site rhuthmos.eu)

Henri Meschonnic, Critique du rythme. Anthropologie historique du langage, Lagrasse, Verdier, 1982

Henri Meschonnic, Politique du rythme, politique du sujet, Lagrasse, Verdier, 1995 cracy and the Social Acceleration of Time, Baltimore and London, Johns Hopkins University Press, 2004

William E. Scheuerman \& Hartmut osa, High-Speed Society: Social Acceleration, Power and Modernity, University Park, Penn State Press, 2009 